Phys. Med. Biol. 41 (1996) 1045-1058. Printed in the UK

\title{
Investigation of a phase-only correlation technique for anatomical alignment of portal images in radiation therapy
}

\author{
Jia-Zhu Wang $\nmid \S$, Lawrence E Reinstein $\dagger$, Joseph Hanley $\ddagger$ and \\ Allen G Meek $\dagger$ \\ $\dagger$ Department of Radiation Oncology, University Hospital, SUNY at Stony Brook, Stony Brook, \\ NY 11794, USA \\ $\ddagger$ Memorial Sloan-Kettering Cancer Center, New York, NY 10021, USA \\ Received 3 October 1995, in final form 9 February 1996
}

\begin{abstract}
A new image registration algorithm based on phase-only correlation is applied to portal images in radiation therapy to detect translational shift. The phase-only correlation shows a sharp peak in the correlation distribution as compared to the broad peak computed from conventional correlation using fast Fourier transform. In this paper, the algorithm of phaseonly correlation is described and its applicability and robustness are tested when applied to portal images used in clinical radiation oncology. The results achieved give evidence that the phase-only correlation will deliver an alternative approach for image registration and image comparison, that may be applicable in routine clinical practice.
\end{abstract}

\section{Introduction}

When a patient is accepted for radiation therapy based on previous diagnostic studies, not only is it important to choose a proper field and setup for administering the prescribed amount of radiation to the tumour volume, but also it is critical to reproduce the treatment setup daily as planned. In practice, in addition to using immobilization devices to minimize patient movement, $\mathrm{x}$-ray films (portal films) exposed on the exit side of the patient are used as a tool to evaluate the treatment and also as evidence that radiation has been delivered to the desired place. If discrepancies are found between the simulator (planning) films and the portal films, a revision of the treatment procedure may be recommended. Although visual comparisons of the portal film with the simulator film, and of the portal film with subsequent portal films, are still standard practice as part of routine clinical evaluation, computerized analysis of these images has recently attracted the interest of both medical physicists and physicians alike. This interest has been spurred on by the development of effective film digitizers, as well as electronic portal imaging devices (EPIDs) which are capable of recording and displaying the digital images for on-line inspection (van Herk and Meertens 1988, Shalev et al 1989, Munro et al 1990, Wong et al 1990, Morton et al 1991, Boyer et al 1992). However, the development of new techniques in field placement analysis for fast evaluation in order to assist automatic setup remains to be a challenge (Shalev et al 1991, Evans et al 1993, Reinstein et al 1992, Ezz et al 1992, Balter et al 1993, Michalski et al 1993). Matching the portal image to the reference image by field edges (Bijhold et al

$\S$ Present address: Department of Radiation Oncology, Long Island Jewish Medical Center, New Hyde Park, NY 11040, USA. 
1991, 1992, Gilhuijs and van Herk 1993, Leszczynski et al 1993), by anatomical structures (Meertens et al 1990, Graham et al 1991, Evans et al 1992, Wang and Fallone 1994), or by a combination of both (van Herk et al 1993), using a variety of mathematical methods, are some of the approaches that have been used.

Correlation is a technique used in quantitative comparison of two functions. Spatial correlation formed on the pixel-by-pixel level is one of the methods used for image registration. Several groups have investigated field verification in radiation therapy in terms of a normalized correlation computed using the fast Fourier transform (FFT) algorithm (Jones and Boyer 1991), or simply in the spatial domain (Moseley and Munro 1994). Some other 'correlation' methods for field matching include comparison of the curvatures of two open curves (Balter et al 1992) and computing pseudocorrelation using $\chi^{2}$ statistic (Radcliffe et al 1994).

The conventional correlation, computed either in the spatial domain or in the Fourier space, has a rather broad distribution-making the detection of the peak difficult (Jones and Boyer 1991). In this paper, a different approach is employed: the phase information in the Fourier transform of an image is emphasized and the correlation of a reference image and a test image is carried out with the magnitude of the complex spectra in one of the images entirely suppressed. The distribution from the phase-only correlation (POC) shows a sharp peak such that a more accurate maximal correlation can be achieved in determining the translational shift. Chen et al (1994) have demonstrated the feasibility of this approach to correlation of diagnostic images.

In order to introduce this technique and to explore its potential when applied to image registration in radiation therapy, the POC algorithm is applied to digitized simulator and portal images for POC (Wang et al 1995). Results from POC are compared with the distribution from the conventional normalized correlation using FFT technique (CFC, conventional Fourier-based correlation). Simulations when either noise or a small rotation error is present are also carried out.

\section{Theory and method}

\subsection{Conventional correlation}

In image processing, a two-dimensional image $\boldsymbol{g}$ can be described by an array of numbers $g(x, y)$ representing the grey level at different positions $(x, y)$. The normalized crosscorrelation between two 2D images $\boldsymbol{g}$ and $\boldsymbol{h}$ can be defined as

$$
\begin{aligned}
\operatorname{Corr}_{g, h}(a, b)= & \left(\int_{-\infty}^{\infty} \int_{-\infty}^{\infty}[g(x+a, y+b)-\hat{g}][h(x, y)-\hat{h}] \mathrm{d} x \mathrm{~d} y\right) \\
& \times\left[\iint[g(x+a, y+b)-\hat{g}]^{2} \mathrm{~d} x \mathrm{~d} y\right]^{-\frac{1}{2}}\left[\iint[h(x, y)-\hat{h}]^{2} \mathrm{~d} x \mathrm{~d} y\right]^{-\frac{1}{2}}
\end{aligned}
$$

where ' ' indicates the mean grey level in each image. The normalized correlation has a 2D spatial distribution with arguments $a$ and $b$ representing the spatial shift of one image relative to the other. In the discrete case, the correlation distribution can be treated as a matrix with elements ranging from -1 to +1 .

Taking two images A and B in figure 1 as an example, when the test image B is shifted relative to the reference image A such that similar patterns overlap, the correlation computed by equation (1) becomes a maximum. At the location where maximum correlation occurs, an arrow connecting the centres of the two images indicates the size and direction of the shift between the patterns, as shown in the lower panel of figure 1 . Therefore, by finding the 

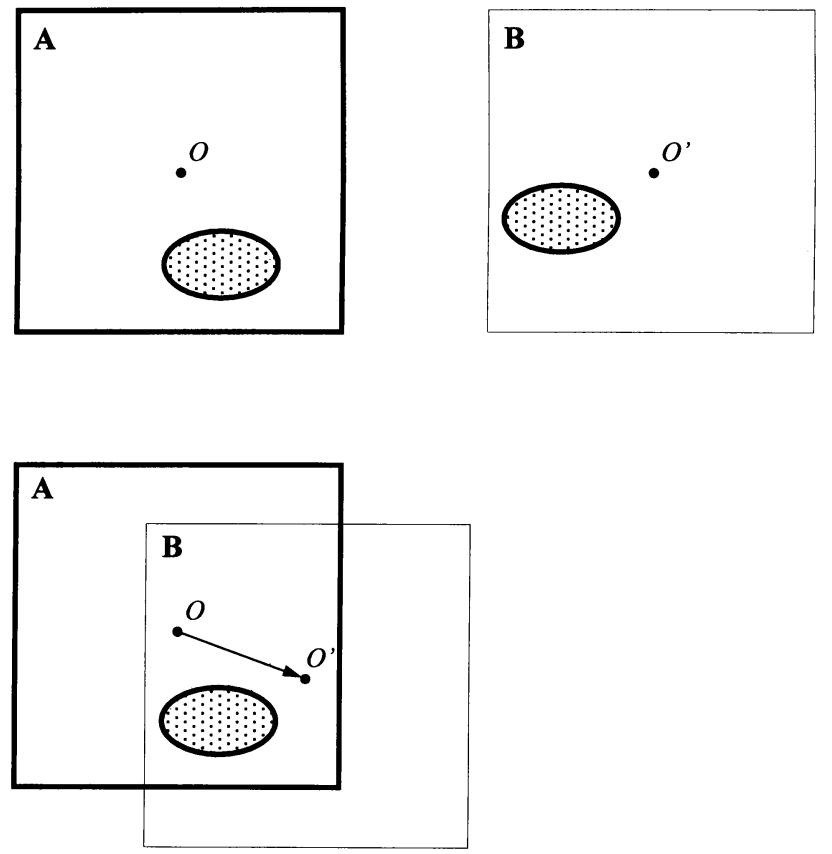

Figure 1. A schematic drawing of a reference image A and a test image B. Lower panel, the correlation becomes maximum when the patterns in A and B overlap, where the arrow indicates the translation shift.

peak position in the correlation distribution, one can detect the translation shift of one image relative to the other based on the internal patterns. Similarly, positions of the correlation peak of portal with portal and portal with simulator images indicate possible patient shifts based on anatomical structure.

It is very time consuming to compute the whole correlation matrix at different shifts when large image files are concerned, even with the help of modern computers. Hence, FFT is employed for accomplishing the task. An image can be described in the frequency domain by complex values

$$
G(u, v)=\int_{-\infty}^{\infty} \int_{-\infty}^{\infty} g(x, y) \mathrm{e}^{-\mathrm{i}(x u+y v)} \mathrm{d} x \mathrm{~d} y
$$

where $u$ and $v$ are the frequency components along the $x$ and the $y$ directions. The Fourier transform $G(u, v)$ of an image $\boldsymbol{g}$ describes the spatial variation rate of grey levels, with each frequency as a complex number consisting a magnitude and a phase.

The correlation becomes a simple multiplication in the frequency domain

$$
G(u, v) H(u, v)^{*}
$$

where $H(u, v)$ is the Fourier transform of image $\boldsymbol{h}$ and ${ }^{*}$ denotes the complex conjugate so that the numerator of the normalized correlation matrix in (1) can be derived as the inverse Fourier transform of (3). The two normalization factors, referred to as $G_{\text {norm }}$ and $H_{\text {norm }}$ hereafter, in the denominator of (1) can also be computed in the frequency domain (Jones 
and Boyer 1991). Therefore, the normalized CFC is given by

$$
\begin{aligned}
\mathrm{CFC}_{g, h}(a, b) & =\frac{F^{-1}\left\{G(u, v) H(u, v)^{*}\right\}}{G_{\text {norm }}(a, b) H_{\text {norm }}} \\
& =\left(\frac{1}{(2 \pi)^{2}} \int_{-\infty}^{\infty} \int_{-\infty}^{\infty} G(u, v) H(u, v)^{*} \mathrm{e}^{\mathrm{i}(x u+y v)} \mathrm{d} u \mathrm{~d} v\right) / G_{\text {norm }}(a, b) H_{\text {norm }} .
\end{aligned}
$$

\subsection{Phase-only correlation}

In general both the magnitude and the phase are needed to completely describe a function in the frequency domain. Sometimes, only information regarding the magnitudes is displayed, such as in the power spectrum, where phase information is completely discarded. However when the relative roles played by the phase and the magnitude in the Fourier domain are examined, it is found that the phase information is considerably more important than the magnitude in preserving the features of an image pattern. Oppenheim and Lim have proven this by reconstructing images using the full information from the phase with unit magnitude. This gave rise to images which resemble the originals in contours of the patterns. On the other hand, the Fourier synthesis using full-magnitude information with a uniform phase resulted in nothing meaningful as compared to the original images (Oppenheim and Lim 1981). Inspired by the above findings, investigations of the use of phase-only information for matched filters or pattern recognition have been carried out. It is found that the phaseonly approach produces a sharper correlation peak (Horner and Gianino 1984a, b).

The phase-only correlation of image $\boldsymbol{g}$ and $\boldsymbol{h}$ is defined as

$$
\operatorname{POC}_{g, h}(a, b)=F^{-1}\left\{G(u, v) H_{\phi}(u, v)^{*}\right\} / G_{n o r m}(a, b) .
$$

Here, a partial normalization factor $G_{n o r m}$, the same as that used in (4), is employed to compute the POC analogous to the normalized CFC in order to compare the two techniques on common ground. $H_{\phi}(u, v)$ is the phase-only Fourier transform of image $\boldsymbol{h}$, in which the magnitude is unity throughout the spectrum, given by

$$
H_{\phi}(u, v)=H(u, v) /|H(u, v)| \text {. }
$$

Hence, from (6), one can take another point of view and consider the reciprocal of $|H(u, v)|$ as a very complicated high-pass filter, since most real objects have Fourier spectra which fall off with increasing frequency (Horner and Leger 1985). High-pass pre-filtering is very effective for differentiating subtle patterns and emphasizing the edge information.

\subsection{Relative significance ratio of correlation peak}

Figure 2 is a typical example of profiles along the horizontal and vertical lines passing through the correlation peak. The top panels show the profiles from the normalized CFC, the bottom panels those from POC. The dashed line in each figure represents the baseline, which is taken as the mean value of the entire distribution. Note that all the peaks are normalized to the same height in this display, so that the absolute values do not play an important role here. It is the ratios of peak height against peak width that are of interest to us since they represent a measure of the selectivity of the correlation.

While it is difficult to compare the success or the failure of CFC and POC due to the differences in scalings, peak widths, and baseline levels, we attempt to develop a scale invariant measure to quantify the comparison. First, the full width at half maximum (FWHM) is introduced in each profile with the peak height measured from the baseline. As 
Horizontal Profile
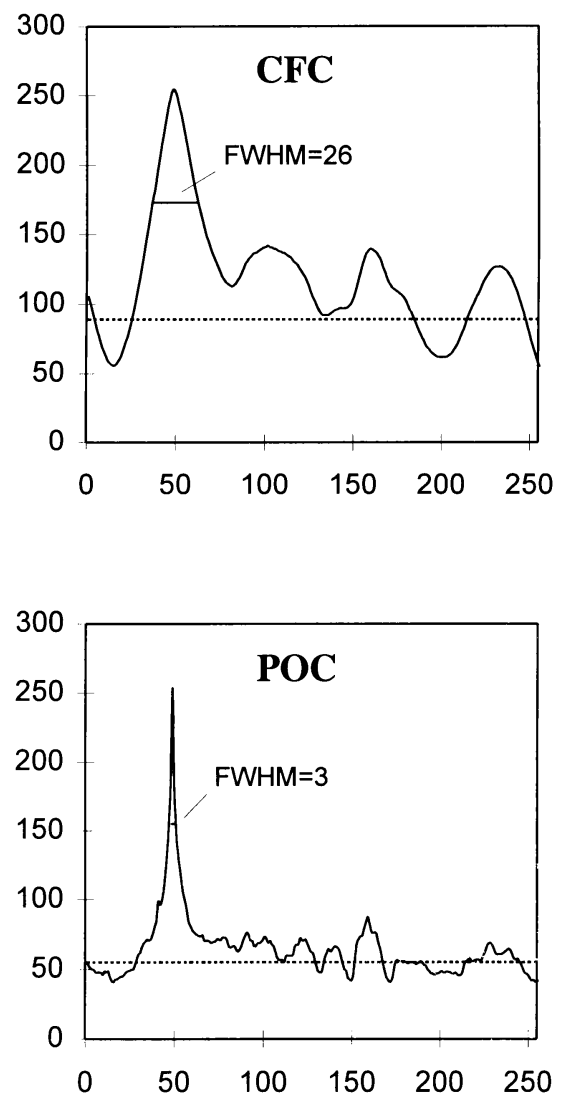

\section{Vertical Profile}
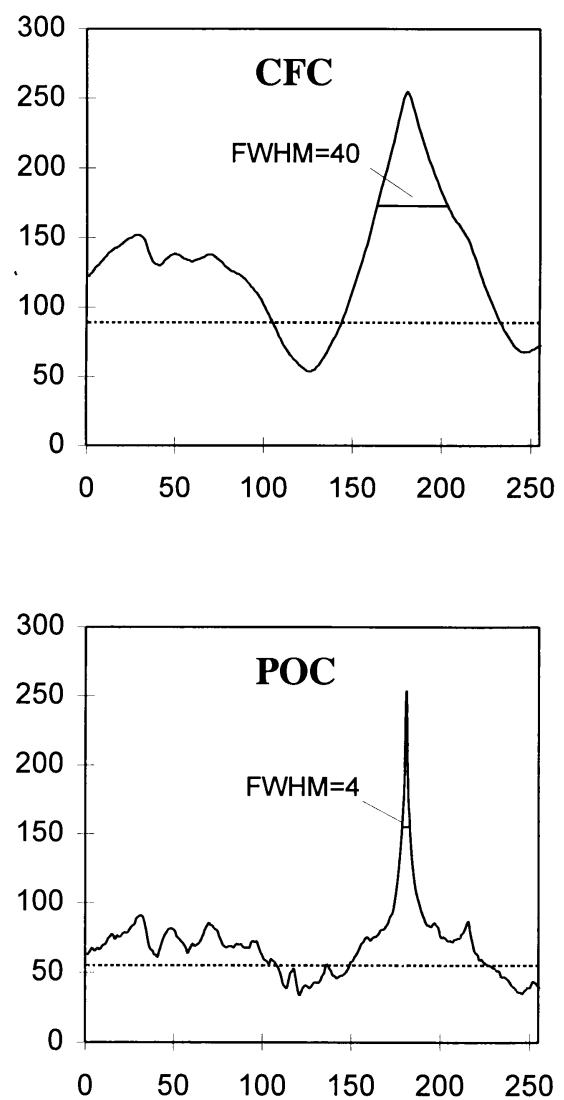

Figure 2. Profiles of the correlation distributions as seen along the horizontal and vertical lines passing through the peak. The dashed lines are the baselines which represent the background level in each correlation distribution. FWHMs are represented in terms of the pixel size along each profile.

shown in figure 2, the FWHM of the profiles given by the number of the pixels in POC is about one-ninth of that from $\mathrm{CFC}$ on average.

In order to consider the two-dimensional nature of the correlation peak, we further introduce a mean FWHM of the peak

$$
\langle\mathrm{FWHM}\rangle=\sqrt{\text { total number of pixels at half maximum. }}
$$

Therefore, the $\langle\mathrm{FWHM}\rangle$ may be analogous to the 'RMS diameter' of the cross section at the half maximum of the peak measured from the baseline. In order to provide a measure to the correlation peak against the peak sharpness, a relative significance ratio $(R)$ of the correlation peak is introduced as

$$
R=\langle\mathrm{FWHM}\rangle^{-1}\left(y_{\max }-\bar{y}\right) /\left(\bar{y}-y_{\min }\right)
$$

where $y_{\max }$ is the peak height, $\bar{y}$ the baseline, and $y_{\min }$ the minimum in the correlation distribution. The denominator $\bar{y}-y_{\min }$ is introduced into the definition so that the significance ratio is scale invariant. Hence a larger value of $R$ describes a higher or a 
sharper correlation peak, making the correlation more selective. A program was developed in $\mathrm{C}$ language to compute $\langle\mathrm{FWHM}\rangle$ and the relative significance ratio in each correlation distribution was computed. For the results in figure $2, R_{C F C}=0.05$ in CFC, and $R_{P O C}=0.8$ in POC.

\section{Testing and results}

\subsection{Computer algorithm}

The algorithm for computing the CFC was developed following the references of Jones and Boyer (1991) and others (Pratt 1991, Pitas 1993). Additional references are used for the POC algorithm (Horner and Gianino 1984a, b). The software was written in C on an HP 730 workstation running HP-UX 9.0. Computation of the normalized correlation, which involves FFT of two images in the numerator, two in the denominator, and two inverse FFTs takes approximately $40 \mathrm{~s}$.

In order to define the value of correlation when a portion of the test image extends beyond the border of the reference image, the image matrices are embedded into larger matrices padded with zeros before computing the correlation (Pitas 1993).

\subsection{Correlation between portal images}

In order to carry out a systematic investigation, different portal films were selected and digitized using a scanning laser digitizer $\dagger$. The reference image $(200 \times 200)$ in figure 3(a) is a pelvic field from a patient, where the exit beam from the patient forms extremely lowcontrast images with limited anatomical detail. The test image $(80 \times 80)$ in figure $3(\mathrm{~b})$, enclosing part of the pubic bone and obturator foramen, is a subregion of the reference image in this study so that the direction and the amount of the shift $(48,48)$ are known and serve as the criteria to test the POC technique. The numbers, obtained by comparing the centres of the two images, indicate that the test image is shifted 48 pixels to the right, 48 pixels to the inferior of the reference image.

As explained in section 2, the correlation coefficient is a two-dimensional function in the spatial domain and can be rescaled to range from zero to 255 in order to be displayed as an 8 bit raw image for further evaluation. The whitest spot corresponds to the maximum of the correlation and the darkest spot the minimum. The position of the peak indicates the translational shift between the test and the reference images.

Although results from both methods give the correct peak location $(48,48)$ which matches the known result, the peak of CFC is broader, as shown in figure 3(c), while the peak of POC is much sharper and appears as a white dot on a mainly dark background (figure 3(d)). An arrow renders assistance to locate the peak in POC for fear that the white spot might be too small to be noticed visually. The relative significance ratios are calculated to be $R_{C F C}=0.11$ and $R_{P O C}=4.91$.

Figure $4($ a) is a pelvic image $(240 \times 240)$ of another patient, which is of an overall poorer contrast. The test image $(100 \times 100)$ shown in figure $4(b)$ is a subregion of figure 4(a). Contrast has been enhanced in the original images for display only. The correlation distribution from CFC results in a broad peak as shown in figure 4(c) with the relative significance ratio $R_{C F C}=0.09$, whereas the distribution from POC gives rise to an extremely sharp peak that looks almost like a pin hole (figure 4(d)) with $R_{P O C}=12.18$.

$\dagger$ Lumisys Lumiscan 50. 


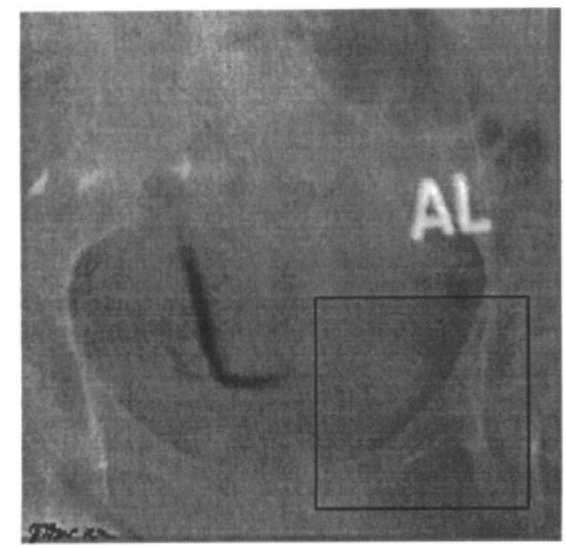

(a)

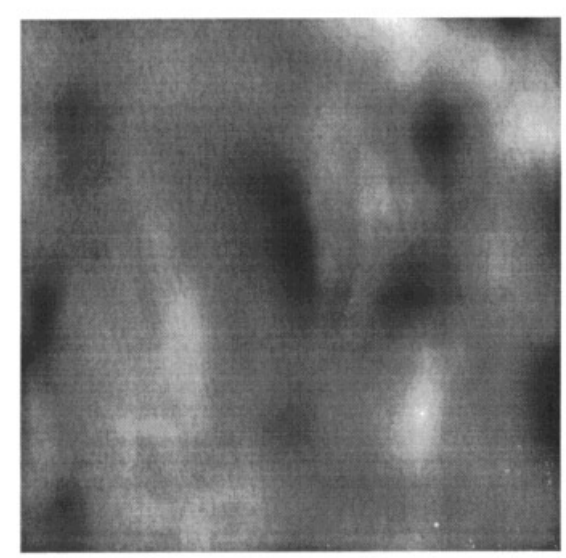

(c)

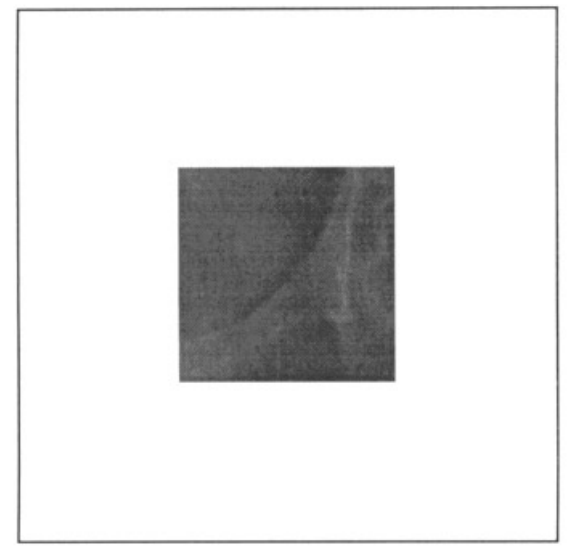

(b)

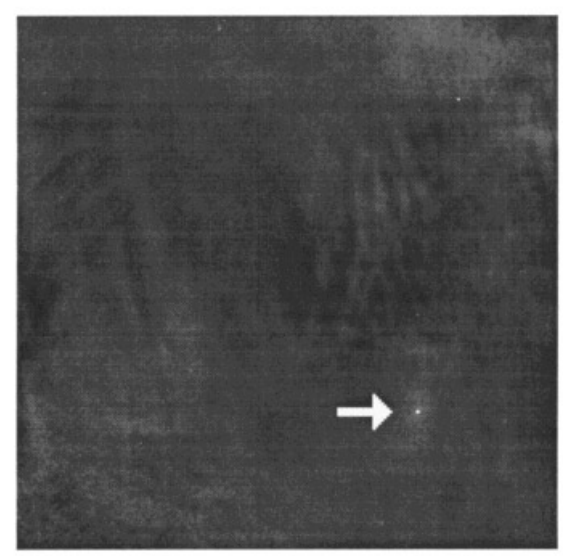

(d)

Figure 3. (a), (b) Two images from a digitized portal film and its subregion. (c) A grey scale display of correlation function of the two images computed with normalized CFC. The result is rescaled and always ranges from zero to 255. (d) A correlation distribution of the two images computed with POC. A white arrow indicates the position of the correlation peak.

Several thin lines in figure 4(d) are the results of correlation when the edges of the two images aligned with each other.

\subsection{Results in the presence of added noise or rotation error}

To further test the algorithm, random noise was added to the test images. The noise is assumed to have a Gaussian distribution. Thus, every pixel of the test image is superimposed with a random noise value, either positive or negative. The level of noise is defined as the ratio of the standard deviation of the Gaussian distribution to the peak-to-peak grey level in each image. Random noise with $20 \%$ noise level is added to the test image in figure 3(b), and $30 \%$ noise to figure 4 (b). The resulting images with noise added are shown in figure 5(a) 


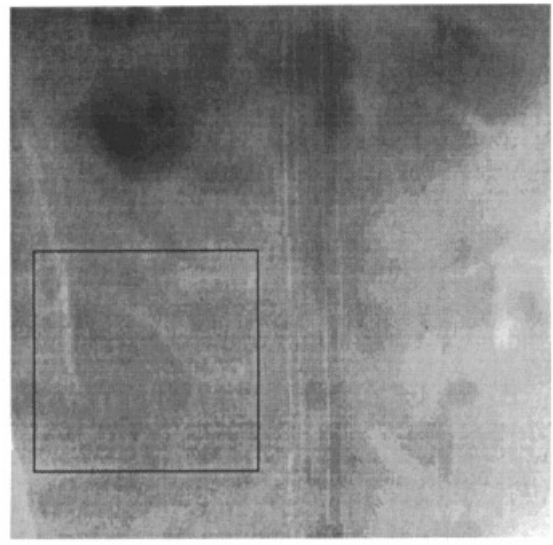

(a)

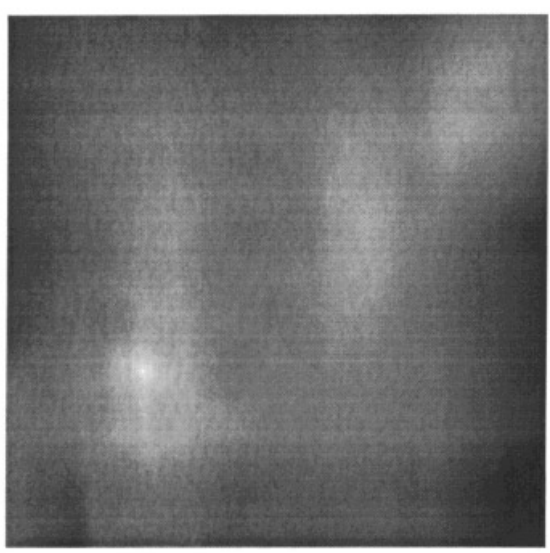

(c)

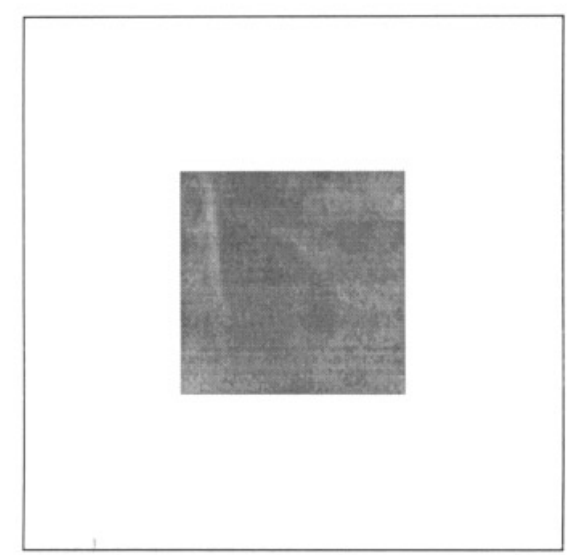

(b)

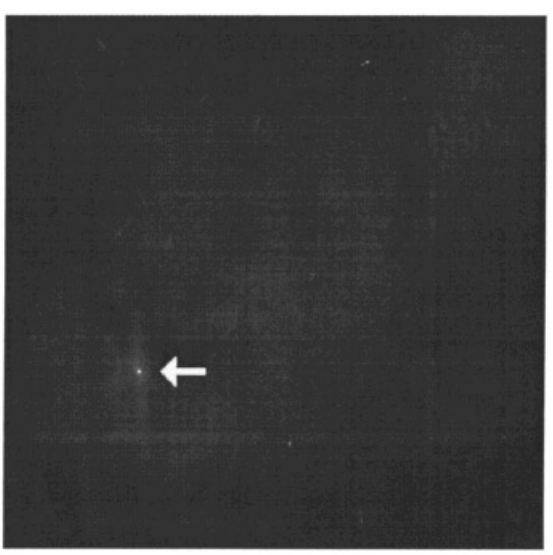

(d)

Figure 4. (a) The reference image. (b) The test image is a subregion of the reference image. (c) A correlation distribution from normalized CFC. (d) A correlation distribution from POC.

and (c). The results of POC of figure 5(a) with figure 3(a), and figure 5(c) with figure 4(a), are given in figure 5(b) and (d) respectively. As compared with the noise-free cases in figure 3(d) and 4(d), the peak positions are still quite stable, although the background is observed to increase. The distributions computed from CFC are not shown here but their peak profiles will be discussed later. In general, the peaks in CFC are still much broader than those in POC when noise is present, and some of the local peaks in CFC become higher $\left(R_{C F C}=0.10, R_{P O C}=0.76\right.$ in figure $5(\mathrm{~b}) ; R_{C F C}=0.09, R_{P O C}=1.26$ in figure 5(d)). However, the change in overall background in CFC is less sensitive to noise as compared to POC.

In practice patient rotations, either in plane or out of plane, will cause errors in the patient setup. To detect the rotational error, especially to provide a quantitative measure, is an extremely difficult task. More sophisticated techniques are involved to resolve this issue, such as the use of Fourier-Mellin invariant descriptors (Chen et al 1994). In this paper, as 


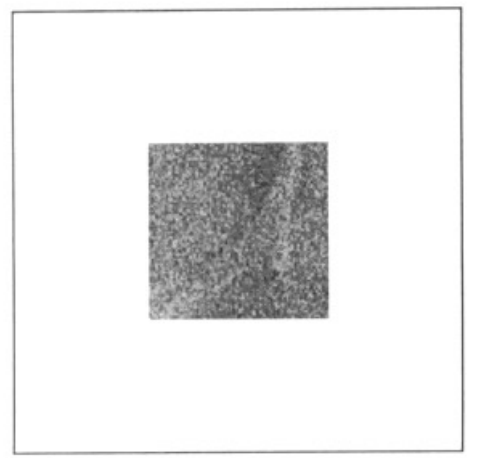

(a)

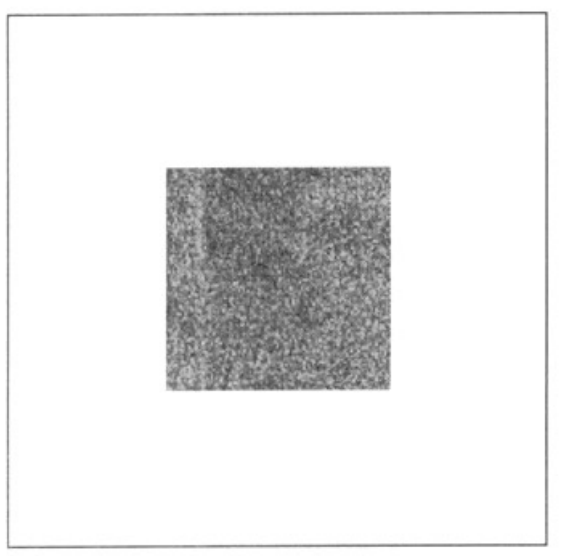

(c)

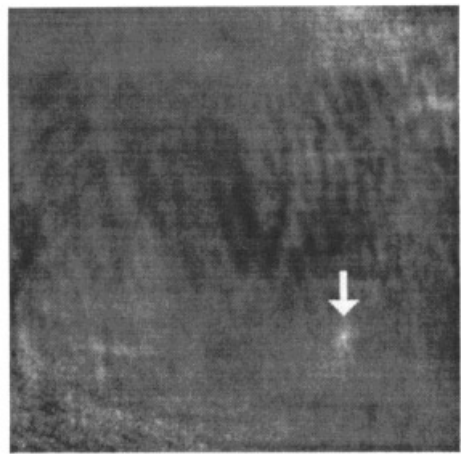

(b)

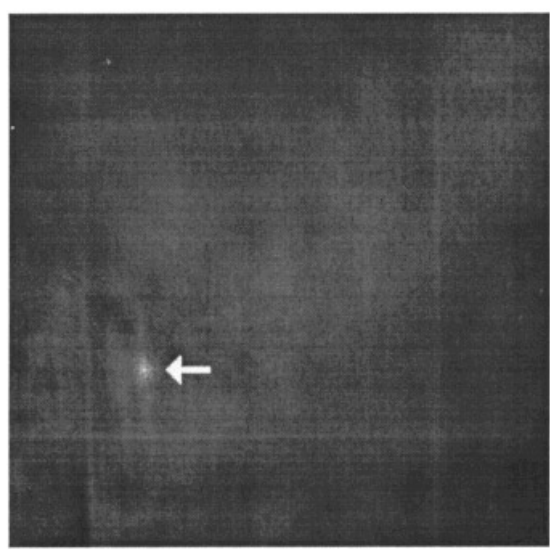

(d)

Figure 5. (a) $20 \%$ noise added to the image in figure 3(b). (b) POC of images in figure 5(a) and figure 3(a). (c) $30 \%$ noise added to the image in figure 4(b). (d) POC of images in figure 5(c) and figure $4(\mathrm{a})$.

a preliminary study, we would like to address the ability of the POC technique to detect translational shifts under conditions of small rotational errors.

First, the test image in figure $3(\mathrm{~b})$ is rotated by $2^{\circ}$ around the centre. The correlation of this rotated test image with the reference image (figure 3(a)) is computed. The results from CFC and POC are shown in figure 6(a) and (b), respectively. Figure 6(c) and (d) are the correlation results of similar situations as (a) and (b) but with a rotation error of $4^{\circ}$. In the case of POC, the peak position is $(48,47)$ when the rotation error is $2^{\circ}$, and $(46$, 47) when the rotation error is $4^{\circ}$. In each case of CFC, there is a higher peak at the upper boundary, as shown in figure 6(a) and (c). Apart from those peaks near the boundary, the secondary peaks in the CFC have the same positions as those in POC. The background noise in both correlation distributions becomes higher when the angle of rotation becomes larger. Irrespective of the increasing background, the peak from POC is still sharper than the peak from CFC. The relative significance ratio $R_{C F C}=0.06, R_{P O C}=0.32$ when rotation error 


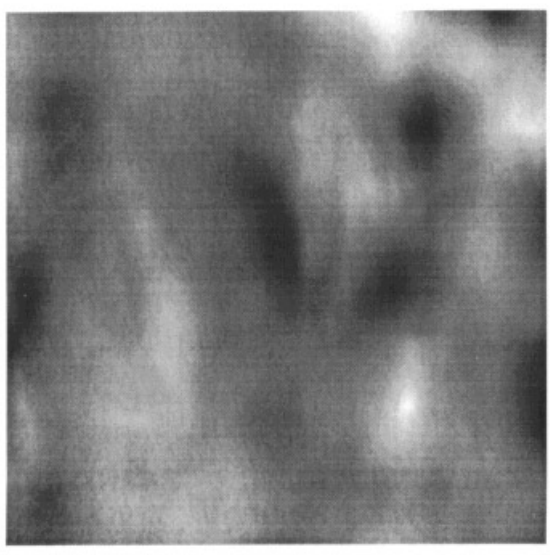

(a)

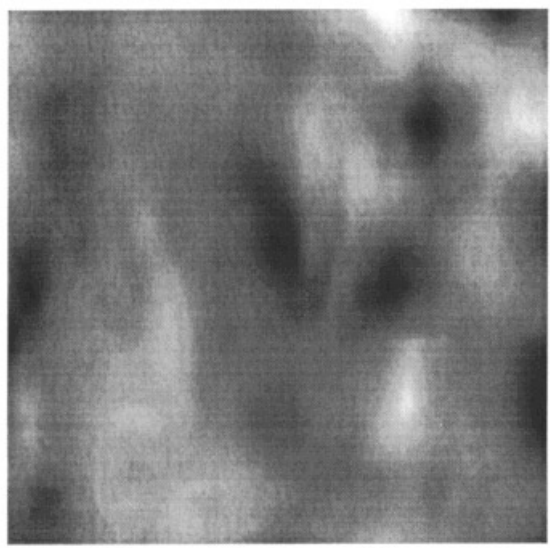

(c)

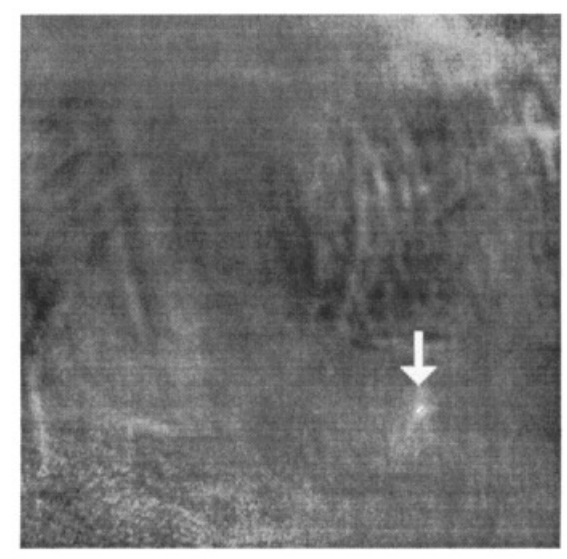

(b)

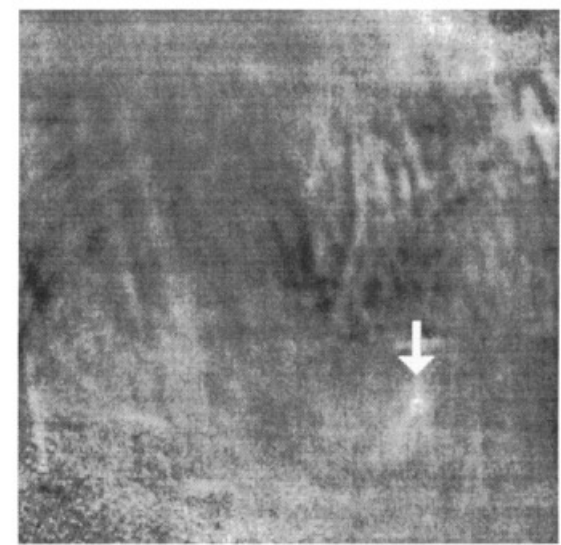

(d)

Figure 6. (a) Normalized CFC of figure 4(a) and (b) (with the latter rotated in plane by $2^{\circ}$ ). (b) POC of the same images as used in (a). (c) Normalized CFC of figure 4(a) and (b) (rotated by $4^{\circ}$ ). (d) POC of the same images as used in (c).

is $2^{\circ}$, and $R_{C F C}=0.06, R_{P O C}=0.10$ when error is $4^{\circ}$. Hence, it may be prudent to conclude that within a limit of a few degrees of rotation error $\left(<4^{\circ}\right)$, the peak position of POC can give rise to the correct translational shift.

\subsection{Correlation between phantom images}

We next turn to the phantom studies. A solid anthropomorphic pelvic phantom $\dagger$ was positioned at different known translational distances and portal films were acquired. The films were digitized and two images, each with $190 \times 190$ pixels, are shown in figure 7(a) as the reference image and 7 (b) as the test image. A region of $70 \times 70$ pixels was selected

$\dagger$ The Phantom Laboratory, Salem, NY, USA. 


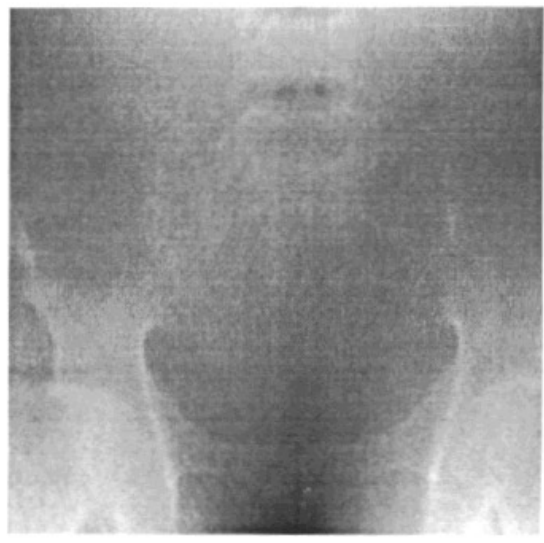

(a)

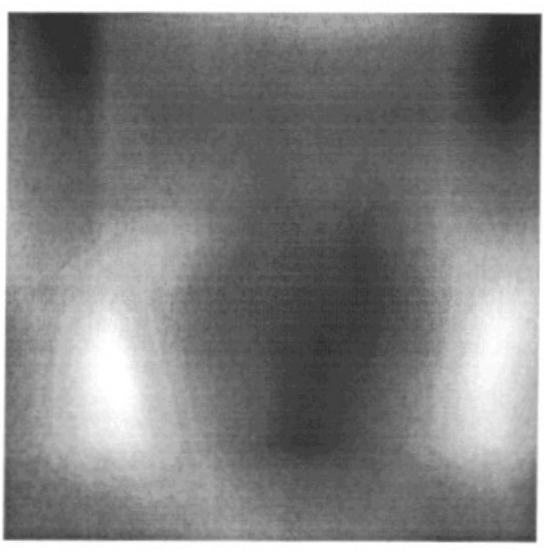

(c)

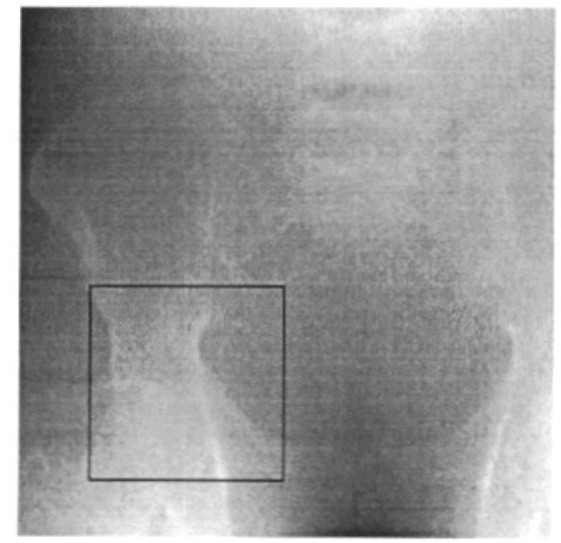

(b)

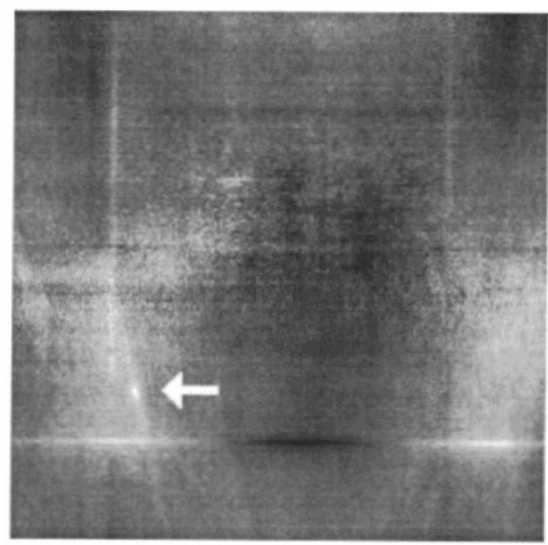

(d)

Figure 7. (a), (b) Two digitized portal images of a pelvis phantom at different positions. (c) The normalized CFC of (a) and (b) displays two broad peaks. (d) The POC distribution displays a correlation peak, together with edge effects.

from figure 7(b) as indicated by a small frame.

The correlation computed from CFC showed two broad peaks (figure 7(c)). The peak on the left of the image is slightly higher than that on the right and indicates the phantom shift $1.9 \mathrm{~cm}$ to the right, and $0.8 \mathrm{~cm}$ inferior. Correlation from POC resulted in a peak as shown in figure 7(d), which indicated that the test image is shifted $1.6 \mathrm{~cm}$ right laterally and $0.1 \mathrm{~cm}$ inferiorly with respect to the reference image. The actual shift was $1.65 \pm 0.05 \mathrm{~cm}$ right laterally and $0 \pm 0.05 \mathrm{~cm}$ along the caudad-to-cephalad direction. The edge effect appears stronger here mainly because the correlation peak is relatively lower. As mentioned earlier, these line artifacts are the loci of the centre of the test region when its edge aligns with one side of the reference image. These artifacts can be removed according to the size of the test region. 
Fig. Ref. $\quad \mathrm{R}_{\mathrm{CFC}} \quad \mathrm{R}_{\mathrm{POC}} \quad$ Horiz. Profile $\quad$ Vert. Profile

(a) $3 c \& 3 d$

0.11

4.91

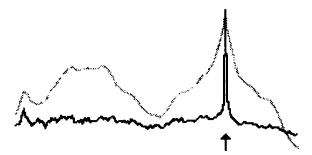

(b) $4 \mathrm{c} \& 4 \mathrm{~d}$

0.09

12.18
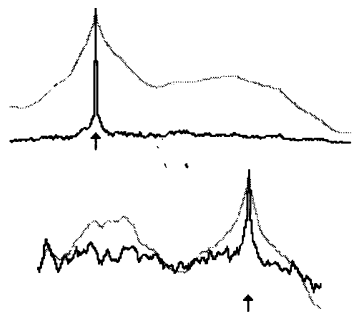

(c) $5 \mathrm{~b}$

0.10

0.76

0.09

(d) $5 \mathrm{~d}$

(e) $6 a \& 6 b$

0.06

0.32

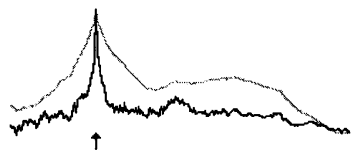

1.26

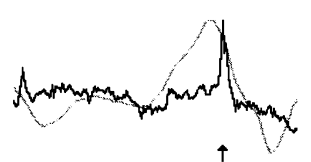

(f) $6 \mathrm{c} \& 6 \mathrm{~d}$

0.06

0.10

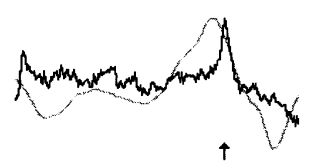

(g) $7 \mathrm{c} \& 7 \mathrm{~d}$

0.03
0.08
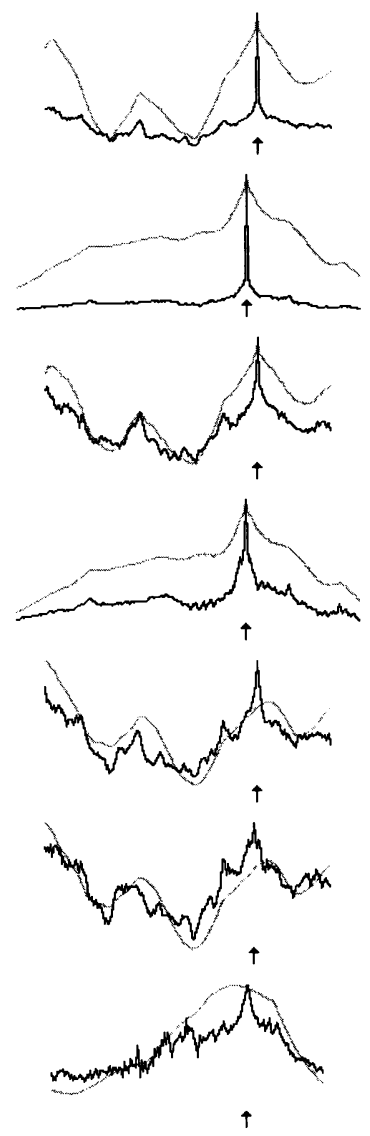

Figure 8. Left panels, relative significance ratio $R$ calculated from CFC and POC; middle and right panels, horizontal and vertical profiles taken through the correlation peaks for the cases studied in figures 3-7. Thicker curves represent results from POC and thinner curves those from the normalized CFC. The small arrow indicates the correct peak position as anticipated from known parameters in each case.

Figure 8 summarizes the results and consists of a list of profiles that pass through the highest peak in each correlation distribution as discussed above. The left panels show the profiles along the horizontal line and the right panels the profiles along the vertical line. The corresponding figure numbers, from which profiles are derived, are listed for the reader's reference. Results computed from POC are shown as thicker curves, while results from CFC are shown thinner. The profiles of POC in figure $8(\mathrm{~g})$ are shown with edge effect removed. A small arrow in each case indicates the correct peak position as anticipated. Both peaks from CFC and POC match the anticipated values in figures 3-5. The peak from POC is markedly sharper than that obtained from CFC. The relative significance ratios are also summarized and listed together with the profiles. 


\section{Discussion}

We have reported on the algorithmic development of the phase-only correlation (POC), as well as the tests of its applicability to radiation therapy. The results of the tests suggest that POC may provide an alternative image registration method for detecting translational shifts. As these were only preliminary tests, our study was limited and included several simplifications: possible advantages of image preprocessing were not considered and robustness under small out-of-plane rotations has not yet been considered. To test its applicability to routine clinical practice, more phantom studies should be carried out in the next phase of this investigation.

In order to explore the validity and limitations of the method itself, no image preprocessing was applied to the images before carrying out the POC (except for the preprocessing inherent to the method itself or the film digitizer). The same approach was also adopted when using the normalized CFC technique. Image preprocessing has been shown to enhance the performance of CFC (Pratt 1991). Therefore, it may be worthwhile to investigate whether image preprocessing can improve the results of POC.

Note that in (5), the phase information is only extracted from the test image and not from the reference image in computing the POC. Alternatively, POC was carried out by extracting the phase information from the reference image and not the test image. In general, similar results were observed. Furthermore, correlations were tested when phase information was extracted from both images. Again, similar results were observed for the images studied.

\section{Conclusions}

The phase-only procedure provides a new technique for the correlation of treatment fields in radiation therapy. Several preliminary tests investigated in this paper have demonstrated that the POC method provides an alternative to conventional correlation techniques (CFC) which may be advantageous with respect to the sharpness of the correlation peak. Although background level in POC is sensitive to the presence of noise and the rotation errors, the position and the sharpness of correlation peaks appear to be robust in the presence of noise or small in-plane rotation errors.

The experience gained from this study and the results obtained from the simulations and phantom studies enable us to proceed and to investigate its further application to clinical practice: the correlation of portal with portal or portal with simulator images on a daily basis to evaluate the patient setup during the treatment.

\section{Acknowledgments}

The authors thank Drs A Boyer and J Balter for their helpful discussions. We also thank Drs $\mathrm{J}$ Liang and $\mathrm{J}$ Ye for providing their printing software and allowing us to use the HP workstation.

\section{References}

Balter J M, Chen G T Y, Pelizzari C A, Krishnasamy S, Rubin S and Vijayakumar S 1993 Online repositioning during treatment of the prostate: a study of potential limits and gains Int. J. Radiat. Oncol. Biol. Phys. 27 $137-43$

Balter J M, Pelizzari C A and Chen G T Y 1992 Correlation of projection radiographs in radiation therapy using open curve segments and points Med. Phys. 19 329-34 
Bijhold J, Gilhuijs K G A and van Herk M 1992 Automatic verification of radiation field shape using digital portal images Med. Phys. 19 1007-14

Bijhold J, Gihuljs K G A, van Herk M and Meertens H 1991 Radiation field edge detection in portal images Phys. Med. Biol. 36 1705-10

Boyer A L, Antonuk L, Fenster A, van Herk M, Meertens H, Munro P, Reinstein L E and Wong J 1992 A review of electronic portal imaging devices (EPIDs) Med. Phys. 19 1-16

Chen Q S, Defrise M and Deconinck F 1994 Symmetric phase-only matched filtering of Fourier-Mellin transforms for image registration and recognition IEEE Trans. Pattern Anal. Machine Intell. PAM1-16 1156-68

Evans P M, Gildersleve J Q, Rawlings C and Swindell W 1993 Technical note: the implementation of patient position correction using a megavoltage imaging device on a linear accelerator Br. J. Radiol. 66 833-8

Evans P M, Phil D, Gildersleve J Q, Morton E J, Swindell W, Coles R, Ferraro M, Rawlings C, Xiao Z R and Dyer J 1992 Image comparison techniques for use with megavoltage imaging systems Br. J. Radiol. 65 701-9

Ezz A, Munro P, Porter A T, Battista J, Jaffray D A, Fenster A and Osborne S 1992 Daily monitoring and correction of radiation field placement using a video-based portal imaging system: a pilot study Int. J. Radiat. Oncol. Biol. Phys. 22 159-65

Gilhuijs K G A and van Herk M 1993 Automatic on-line inspection of patient setup in radiation therapy using digital portal images Med. Phys. 20 667-77

Graham M L, Cheng A Y, Geer L Y, Binns W R, Vannier W R and Wong J W 1991 A method to analyze two-dimensional daily radiotherapy portal images from an on-line fiber-optic imaging system Int. J. Radiat. Oncol. Biol. Phys. 20 613-19

Horner J L and Gianino P D 1984a Phase-only matched filtering Appl. Opt. 23 812-16

- 1984b Additional properties of the phase-only matched filtering Opt. Eng. 23695

Horner J L and Leger J R 1985 Pattern recognition with binary phase-only filters Appl. Opt. 24 609-11

Jones S M and Boyer A L 1991 Investigation of an FFT-based correlation technique for verification of radiation treatment set-up Med. Phys. 18 1116-25

Leszczynski K W, Shalev S and Gluhchev G 1993 Verification of radiotherapy treatments: computerized analysis of the size and shape of radiation fields Med. Phys. 20 687-94

Meertens H, Bijhold J and Strackee J 1990 A method for the measurement of field placement errors in digital portal images Phys. Med. Biol. 35 299-323; Int. J. Radiat. Oncol. Biol. Phys. 27 1199-1206

Michalski J M, Wong J W, Gerber R L, Yan D, Cheng A, Graham M V, Renna M A, Sawyer P J and Perez C A 1993 The use of on-line image verification to estimate the variation in radiation therapy dose delivery Int. J. Radiat. Oncol. Biol. Phys. 27 707-16

Morton E J, Swindell W, Lewis D G and Evans P M 1991 A linear array, scintillation crystal-photodiode detector for Megavoltage imaging Med. Phys. 18 681-91

Moseley J and Munro P 1994 A semiautomatic method for registration of portal images Med. Phys. 21 551-8

Munro P, Rawlinson J A and Fenster A 1990 A digital fluroscopic imaging device for radiotherapy localization Int. J. Radiat. Oncol. Biol. Phys. 18 641-9

Oppenheim A V and Lim J S 1981 The importance of phase in signals Proc. IEEE 69 529-41

Pitas I 1993 Digital Image Processing Algorithms (London: Prentice-Hall)

Pratt W K 1991 Digital Image Processing 2nd edn (New York: Wiley)

Radcliffe T, Rajapakshe R and Shalev S 1994 Pseudocorrelation: a fast, robust, absolute, grey-level image alignment algorithm Med. Phys. 21 761-9

Reinstein L E, Pai S and Meek A 1992 Assessment of geometric treatment accuracy using time-lapse display of electronic portal images Int. J. Radiat. Oncol. Biol. Phys. 22 1139-46

Shalev S, Ding X, Strachan R and Ryder S 1991 The quantification of radiation treatment accuracy Med. Biol. Eng. Comput. 29 (Supplement) 857

Shalev S, Lee T, Leszczynski K, Cosby S, Chu T, Reinstein L and Meek A 1989 Video techniques for on-line portal imaging Comput. Med. Imaging Graphics 13 217-26

van Herk M, Bel A, Gilhuijs K G A and Vijlbrief R E 1993 A comprehensive system for the analysis of portal images Radiother. Oncol. 29 221-29

van Herk M and Meertens H 1988 A matrix ionization chamber imaging device for on-line patient setup verification during radiotherapy Radiother. Oncol. 11 369-78

Wang H and Fallone G G 1994 A robust morphological algorithm for automatic radiation field extraction and correlation of portal images Med. Phys. 21 237-44

Wang J Z, Reinstein L E, Hanley J and Meek A G 1995 Phase-only correlation applied to radiation therapy Med. Phys. 22991

Wong J W, Binns W R, Cheng A Y, Geer L Y, Epstein J W, Klarmann J and Purdy J A 1990 On-line radiotherapy imaging with an array of fiber-optic image reducers Int. J. Radiat. Oncol. Biol. Phys. 18 1477-84 